\title{
A NOTE ON THE ERGODIC THEOREM
}

\author{
J. R. BLUM ${ }^{1}$ AND D. L. HANSON
}

Let $\Omega$ be a set, $\mathfrak{F}$ a $\sigma$-algebra of subsets of $\Omega$, and $T$ a 1-1 transformation which maps $\Omega$ onto $\Omega$ and is bimeasurable, i.e., $A \in \mathfrak{F}$ if and only if $T A \in \mathfrak{F}$. Let $P$ be a probability measure defined on $\mathfrak{F} . P$ is said to be invariant provided $P(A)=P(T A)$ for all $A \in \mathfrak{F}$. For such probability measures the individual ergodic theorem holds, i.e., if $f$ is a measurable function defined on $\Omega$ such that $\int_{\Omega}|f| d P<\infty$ then $\lim _{n \rightarrow \infty}(1 / n) \sum_{i=0}^{n-1} f\left(T^{i} x\right)$ exists for almost all $x$. Let $B$ be the class of bounded measurable functions defined on $\Omega$. In this note we show that the individual ergodic theorem holds for members of $B$ for a class of probability measures which includes the invariant ones and is considerably wider than that class.

Let $M=\left(m_{i, j}, i, j=0,1,2, \cdots\right)$ be an infinite matrix satisfying:

(i) $m_{i, j} \geqq 0$ for all $i$ and $j$,

(ii) $\sum_{j=0}^{\infty} m_{i, j} \leqq C<\infty$ uniformly in $i$,

(iii) $\lim _{i \rightarrow \infty} m_{i, j}=0$ for all $j$,

(iv) $\lim _{i \rightarrow \infty} \sum_{j=0}^{\infty} m_{i, j}=1$.

Such a matrix $M$ represents a positive regular summation method, and a good deal is known about such methods. In particular, such methods preserve positivity and convergence of sequences. See, e.g., Hardy [4]. We shall call such a method shift-invariant provided for every sequence $\left\{a_{n}\right\}$ of real numbers for which $\lim _{n \rightarrow \infty} \sum_{j=0}^{\infty} m_{n, j} a_{j}=a$ exists and is finite we have $\lim _{n \rightarrow \infty} \sum_{j=0}^{\infty} m_{n, j} a_{j+1}=a$. The $(\mathrm{C}, 1)$ method, i.e. $m_{i, j}=1 /(i+1)$ for $j \leqq i$ and $m_{i, j}=0$ for $j>i$, is an example of a shift-invariant method. We shall say the sequence $\left\{a_{n}\right\}$ is $M$ summable if $\lim _{n \rightarrow \infty} \sum_{j=0}^{\infty} m_{n, j} a_{j}$ exists.

Theorem. Let $P$ be a probability measure defined on $\mathfrak{F}$ and let $M$ be a positive, regular, shift-invariant summation method. If the sequence $\left\{P\left(T^{n} A\right)\right\}$ is $M$-summable for each $A \in \mathfrak{F}$ then the ergodic theorem holds for each $f \in \Theta$.

Proof. For each $n$ and each $A \in \mathfrak{F}$ let $Q_{n}(A)=\sum_{j=0}^{\infty} m_{n, j} P\left(T^{j} A\right)$ and let $Q(A)=\lim _{n \rightarrow \infty} Q_{n}(A)$. Then it follows from known results (see, e.g., Halmos [3, p. 170, Problem 14]) that $Q(A)$ is a probability measure. Also since $M$ is shift-invariant we see that $Q$ is invariant. Now let $A \in \mathfrak{F}$ be such that $Q(A)=0$ and let $B=\bigcup_{i=-\infty}^{\infty} T^{i} A$. Then

Received by the editors January 22, 1964.

${ }^{1}$ Research supported by the National Science Foundation, Grant GP-1816. 
$Q(B)=0$ and $B$ is such that $T^{i} B=B$ for all integers $i$. Hence $P\left(T^{n} B\right)$ $=P(B)$ for all $n$ and, consequently, $P(B)=Q(B)=0$. It follows that $P$ is absolutely continuous with respect to $Q$. Now if $f \in B$ then $\int_{\Omega}|f| d Q<\infty$ and the ergodic theorem holds for $f$ with respect to $Q$. But from the absolute continuity of $P$ with respect to $Q$ we see that the ergodic theorem also holds for $f$ with respect to $P$. The theorem is proved.

Note that if the ergodic theorem holds for each $f \in B$ then it follows from the Lebesgue bounded convergence theorem that the sequence $\left\{P\left(T^{n} A\right)\right\}$ is $(\mathrm{C}, 1)$-summable for each $A \in \mathfrak{F}$. Thus we have

Corollary 1. A necessary and sufficient condition that the ergodic theorem hold for each $f \in B$ is that the sequence $\left\{P\left(T^{n} A\right)\right\}$ be $(C, 1)$ summable for each $A \in \mathfrak{F}$.

Another immediate consequence of the theorem is

Corollary 2. Let $M$ be a positive, regular, shift-invariant summation method. If the sequence $\left\{P\left(T^{n} A\right)\right\}$ is $M$-summable for each $A \in \mathfrak{F}$ then it is also $(\mathrm{C}, 1)$-summable.

Corollary 1 is not new. See, e.g., Dowker [2], and Brunk [1].

\section{REFERENCES}

1. H. D. Brunk, On the application of the individual ergodic theorem to discrete stochastic processes, Trans. Amer. Math. Soc. 78 (1955), 482-491.

2. Y. N. Dowker, Invariant measure and the ergodic theorems, Duke Math. J. 14 (1947), 1051-1061.

3. P. R. Halmos, Measure theory, Van Nostrand, New York, 1950.

4. G. H. Hardy, Divergent series, Oxford Univ. Press, Oxford, 1949.

UNIVERSITY OF NEW MEXICO AND

UNIVERSITY OF MISSOURI 